\title{
Improved Error Bounds for Second-Order Differential Equations With Two Turning Points
}

\author{
F. W. J. Olver* \\ Institute for Basic Standards, National Bureau of Standards, Washington, D.C. 20234
}

(July 13, 1976)

New error bounds are given for approximate solutions of differential equations of the form

$$
d^{2} w / d \zeta^{2}=\left\{u^{2}\left( \pm a^{2}-\zeta^{2}\right)+\psi(u, a, \zeta)\right\} w
$$

in terms of parabolic cylinder functions. Here $\zeta$ is a real variable, $a$ is a bounded real parameter, $u$ is a large positive parameter, and $\psi(u, a, \zeta)$ is a continuous function of $a$ and $\zeta$. Zero values of $a$ are admitted.

Key words: Error bounds; parabolic cylinder functions; turning points; uniform asymptotic approximations; Weber's equation.

\section{Introduction and Summary}

Recently [2] ${ }^{1}$, [3], I developed a uniform asymptotic theory of second-order linear differential equations with two coalescing simple turning points, and applied the results to the associated Legendre equation. Subsequently, in the course of writing a paper on connection formulas for multiple turning points [4] it became clear how to effect some improvements in two of the four cases treated in [2]. The purpose of the present note is to describe these improvements. It will be assumed that the reader is familiar with the results presented in [2], and the same notation will be used except where indicated otherwise.

The next section introduces new auxiliary functions for the solutions of the modified Weber equation. The new form of the general approximation theorem is stated and discussed in the third (and concluding) section.

An application of the results to the approximation of Whittaker functions with both parameters large will be published in due course.

\section{Auxiliary Functions for the Modified Weber Functions}

The modified Weber functions $W(b, x)$ and $W(b,-x)$ are solutions of the differential equation

$$
d^{2} w / d x^{2}=\left(b-\frac{1}{4} x^{2}\right) w .
$$

As in [2], §8.6 we denote by $\sigma(b)$ the smallest positive root of the equation

$$
k^{-1 / 2}(b) W(b, x)=k^{1 / 2}(b) W(b,-x),
$$

\footnotetext{
* Present address: Institute for Physical Science and Technology, University of Maryland, College Park, Md. 20742.
1 Figures in brackets indicate the literature references at the end of this paper.
} 
but we now extend this definition to all real values of the parameter $b$. We already know that

$$
\sigma(b) \sim 2 b^{1 / 2}, \quad b \rightarrow+\infty .
$$

Next, because $\sigma(b)$ is bounded by the smallest positive zero of $W(b, x)$, the expansion (15.7) of [1] shows that ${ }^{2}$

$$
\sigma(b) \rightarrow 0, \quad b \rightarrow-\infty .
$$

In place of the definition [2], (8.15) of the weight function $E(b, x)$, we now define

$$
E(b, x)=\left\{\begin{array}{l}
k^{1 / 2}(b), \quad-\infty<x \leq-\sigma(b), \\
\{W(b,-x) / W(b, x)\}^{1 / 2},-\sigma(b) \leq x \leq \sigma(b), \\
k^{-1 / 2}(b), \quad \sigma(b) \leq x<\infty
\end{array}\right.
$$

Then $E(b, x)$ is continuous for all real values of $b$ and $x$, and is also a nondecreasing function of $x$. Furthermore, denoting $1 / E(b, x)$ by $E^{-1}(b, x)$, we have

$$
E(b,-x)=E^{-1}(b, x) .
$$

Using the above definition of $E(b, x)$ we now define modulus and phase functions for all real values of $b$ and $x$ by the relations

$$
\begin{aligned}
W(b, x) & =E^{-1}(b, x) M(b, x) \sin \theta(b, x), \\
W(b,-x) & =E(b, x) M(b, x) \cos \theta(b, x), \\
W^{\prime}(b, x) & =E^{-1}(b, x) N(b, x) \sin \omega(b, x), \\
W^{\prime}(b,-x) & =E(b, x) N(b, x) \cos \omega(b, x) .
\end{aligned}
$$

Thus when $-\infty<x \leq-\sigma(b)$ we have

$$
\begin{aligned}
M(b, x) & =\left\{k(b) W^{2}(b, x)+k^{-1}(b) W^{2}(b,-x)\right\}^{1 / 2}, \\
\theta(b, x) & =\tan ^{-1}\{k(b) W(b, x) / W(b,-x)\}, \\
N(b, x) & =\left\{k(b) W^{\prime 2}(b, x)+k^{-1}(b) W^{\prime 2}(b,-x)\right\}^{1 / 2}, \\
\omega(b, x) & =\tan ^{-1}\left\{k(b) W^{\prime}(b, x) / W^{\prime}(b,-x)\right\} .
\end{aligned}
$$

Secondly, when $-\sigma(b) \leq x \leq \sigma(b)$ we have

$$
\begin{aligned}
& M(b, x)=\{2 W(b, x) W(b,-x)\}^{1 / 2}, \quad \theta(b, x)=\frac{1}{4} \pi, \\
& N(b, x)=\left\{\frac{W^{\prime 2}(b, x) W^{2}(b,-x)+W^{\prime 2}(b,-x) W^{2}(b, x)}{W(b, x) W(b,-x)}\right\}^{1 / 2}, \\
& \omega(b, x)=\tan ^{-1}\left\{\frac{W^{\prime}(b, x) W(b,-x)}{W^{\prime}(b,-x) W(b, x)}\right\}
\end{aligned}
$$

Lastly, when $\sigma(b) \leq x<\infty$ we have

$$
\begin{aligned}
M(b, x) & =\left\{k^{-1}(b) W^{2}(b, x)+k(b) W^{2}(b,-x)\right\}^{1 / 2} \\
\theta(b, x) & =\tan ^{-1}\left\{k^{-1}(b) W(b, x) / W(b,-x)\right\}, \\
N(b, x) & =\left\{k^{-1}(b) W^{\prime 2}(b, x)+k(b) W^{\prime 2}(b,-x)\right\}^{1 / 2} \\
\omega(b, x) & =\tan ^{-1}\left\{k^{-1}(b) W^{\prime}(b, x) / W^{\prime}(b,-x)\right\} .
\end{aligned}
$$

2 The actual asymptotic form of $\sigma(b)$ as $b \rightarrow-\infty$ can be shown to be $\sigma(b)-\frac{1}{2}|b|^{-1 / 2} e^{\pi b}$, but we shall not make use of this more precise result in the present paper. 
Each of the functions $M(b, x), \theta(b, x), N(b, x)$, and $\omega(b, x)$ is continuous in $b$ and $x$. Reflection properties are given by

$$
\begin{array}{ll}
M(b,-x)=M(b, x), & \theta(b,-x)=\frac{1}{2} \pi-\theta(b, x), \\
N(b,-x)=N(b, x), \quad \omega(b,-x)=\frac{1}{2} \pi-\omega(b, x) .
\end{array}
$$

As $x \rightarrow \pm \infty$

$$
M(b, x) \sim|2 / x|^{1 / 2}, \quad N(b, x) \sim|x / 2|^{1 / 2} .
$$

Compared with [2], $\S 8.6$ and 8.7, the modulus functions $M(b, x)$ and $N(b, x)$ agree with their previous definitions, except when $b<0$ and $-\sigma(b)<x<\sigma(b)$. On the other hand the phase functions agree only in the case of $\theta(b, x)$ when $b \geq 0$ and $x \geq-\sigma(b)$.

\section{Approximation Theorem}

The following theorem replaces both Theorems III and IV of [2]. The definitions of $E, M$, and $N$ are those just given in $\S 2$.

Theorem: Assume that for a $\epsilon[0, \mathrm{~A}], \zeta \in\left(\zeta_{1}, \zeta_{2}\right)$, and each value of the positive parameter $\mathrm{u}$, the function $\psi(\mathrm{u}, a, \zeta)$ is continuous and $\bigvee_{\zeta 1, \zeta 2}(\mathrm{~F})$ is bounded. Then in this region the differential equation

$$
\mathrm{d}^{2} \mathrm{w} / \mathrm{d} \zeta^{2}=\left\{\mathrm{u}^{2}\left( \pm \alpha^{2}-\zeta^{2}\right)+\psi(\mathrm{u}, a, \zeta)\right\} \mathrm{w}
$$

has a continuous solution $\mathrm{w}(\mathrm{u}, \alpha, \zeta)$, with continuous first and second partial $\zeta$ derivatives, given by

$$
\mathrm{w}(\mathrm{u}, a, \zeta)=\mathrm{W}\left( \pm \frac{1}{2} \mathrm{u} a^{2}, \zeta \sqrt{2 \mathrm{u}}\right)+\epsilon(\mathrm{u}, a, \zeta)
$$

where

$$
\begin{aligned}
& \frac{|\epsilon(\mathrm{u}, a, \zeta)|}{\mathrm{M}\left( \pm \frac{1}{2} \mathrm{u} \alpha^{2}, \zeta \sqrt{2 \mathrm{u}}\right)}, \frac{\left|\partial_{\epsilon}(\mathrm{u}, a, \zeta) / \partial \zeta\right|}{(2 \mathrm{u})^{1 / 2} \mathrm{~N}\left( \pm \frac{1}{2} \mathrm{u} a^{2}, \zeta \sqrt{2 \mathrm{u}}\right)} \\
& \leq \mathrm{E}^{-1}\left( \pm \frac{1}{2} \mathrm{u} \alpha^{2}, \zeta \sqrt{2 \mathrm{u}}\right)\left[\exp \left\{\frac{\mathrm{l}\left( \pm \frac{1}{2} \mathrm{u} a^{2}\right)}{(2 \mathrm{u})^{1 / 2}} V_{\zeta, \zeta_{2}}(\mathrm{~F})\right\}-1\right]
\end{aligned}
$$

and

$$
1(\mathrm{~b})=\sup _{\mathrm{x} \in(-\infty, \infty)}\left\{\Omega(\mathrm{x}) \mathrm{M}^{2}(\mathrm{~b}, \mathrm{x}) \cdot\right\}
$$

In this result either the upper sign or the lower sign is to be taken throughout. Upper signs correspond to Theorem III of [2], and lower signs correspond to Theorem IV of [2]. In both cases a numerically satisfactory companion solution to $w(u, a, \zeta)$ may be obtained by applying the theorem to the equation obtained from (3.1) by replacing $\zeta$ by $-\zeta$. The proof of the theorem is similar to the proofs of Theorems I, II, III, and IV of [2], and it is unnecessary to record details.

The new result simplifies the general theory by replacing two theorems by a single theorem. The main advantage, however, is that error bounds are now supplied directly for all real values of $\xi$. In contrast, Theorem III furnishes error bounds only for nonnegative values of $\zeta$; compare [2], §9.4. Theorem IV, however, supplies error bounds for all real values of $\zeta$, and a property of Theorem IV that is not shared by the new theorem is that it is possible to prescribe anywhere in the closure of $\left(\zeta_{1}, \zeta_{2}\right)$ a reference point $\zeta_{0}$ at which $\epsilon(u, a, \zeta)$ and $\partial \epsilon(u, a, \zeta) / \partial \zeta$ both vanish. In consequence, it is conceivable that there may be applications in which Theorem IV is to be preferred. The new theorem should generally be used in place of Theorem III, however.

It should be noted that although the structure of the error bounds (3.3) is independent of the choice of ambiguous sign in (3.1), (3.2), and (3.3), the asymptotic nature of the bounds as $u \rightarrow \infty$ differs in the two cases. This is in consequence of the results

$$
\begin{aligned}
& l(b)=O\left(b^{1 / 3}\right), \quad b \rightarrow+\infty, \\
& l(b)=O(1), \quad b \rightarrow-\infty,
\end{aligned}
$$


which correspond to similar results in $\S \S 9.2$ and 10.2 of [2]. Thus, for example, if $|\psi(u, a, \zeta) / \zeta|$ is independent of $u$ and integrable at $\zeta=-\infty$ and $+\infty$ uniformly with respect to $a$, then by taking the balancing function $\Omega(x)$ in the definition [2], (6.3) of the error-control function $F(u, a, \zeta)$ to be $1+|x|$, we may show that

$$
\epsilon(u, a, \zeta)=E^{-1}\left(\frac{1}{2} u a^{2}, \zeta \sqrt{2 u}\right) M\left(\frac{1}{2} u a^{2}, \zeta \sqrt{2 u}\right) O\left(u^{-2 / 3} \ln u\right),
$$

when upper signs are taken, compared with

$$
\epsilon(u, a, \zeta)=E^{-1}\left(-\frac{1}{2} u a^{2}, \zeta \sqrt{2 u}\right) M\left(-\frac{1}{2} u a^{2}, \zeta \sqrt{2 u}\right) O\left(u^{-1} \ln u\right),
$$

when lower signs are used. The relations (3.7) and (3.8) apply as $u \rightarrow \infty$ and are uniform with respect to $a \epsilon[0, A]$ and $\zeta \epsilon(-\infty, \infty)$; compare [2], §§9.3 and 10.3. Similarly for the derivative $\partial \epsilon(u, a, \zeta) / \partial \zeta$.

This research has been supported in part by the U.S. Army Research Office, Durham, Contract DA ARO D 31 124. 73 G204, and the National Science Foundation, Grant GP 32841X2.

\section{References}

[1] Olver, F. W. J., Uniform asymptotic expansions for Weber parabolic cylinder functions of large orders, J. Res. Nat. Bur. Stand. (U.S.), 63B (Math. and Math. Phys.) 131-169 (1959).

[2] Olver, F. W. J., Second-order linear differential equations with two turning points, Philos. Trans. Roy. Soc. London Ser. A 278, 137-174 (1975).

[3] Olver, F. W. J., Legendre functions with both parameters large, Philos. Trans. Roy. Soc. London Ser. A 278, 175185 (1975)

[4] Olver, F. W. J., Connection formulas for second-order differential equations with multiple turning points, SIAM J. Math. Anal. Vol. 8, pp. 127-154 (Feb. 1977).

(Paper 80B4-455) 